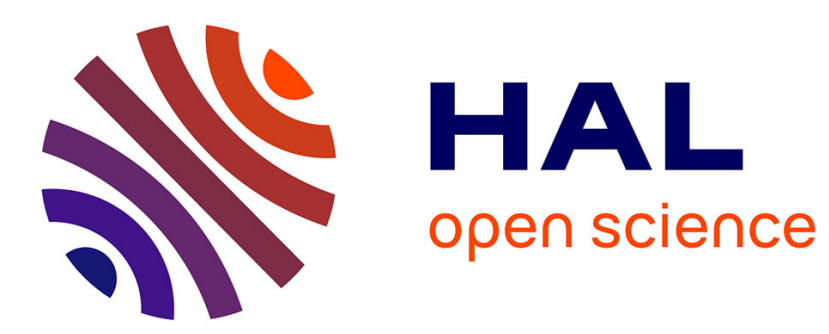

\title{
Investigating the impact of real-time path planning on reducing vehicles traveling time
}

Younes Regragui, Najem Moussa

\section{To cite this version:}

Younes Regragui, Najem Moussa. Investigating the impact of real-time path planning on reducing vehicles traveling time. 2018 International Conference on Advanced Communication Technologies and Networking (CommNet), Apr 2018, Marrakech, France. pp.1-6, 10.1109/COMMNET.2018.8360277. hal-02494903

\section{HAL Id: hal-02494903 \\ https://hal.science/hal-02494903}

Submitted on 29 Feb 2020

HAL is a multi-disciplinary open access archive for the deposit and dissemination of scientific research documents, whether they are published or not. The documents may come from teaching and research institutions in France or abroad, or from public or private research centers.
L'archive ouverte pluridisciplinaire HAL, est destinée au dépôt et à la diffusion de documents scientifiques de niveau recherche, publiés ou non, émanant des établissements d'enseignement et de recherche français ou étrangers, des laboratoires publics ou privés. 


\section{Investigating the Impact of Real-Time Path Planning on Reducing Vehicles Traveling Time}

\author{
Younes Regragui \\ LAROSERI, Department of Computer Science \\ Chouaib Doukkali University, El Jadida Morocco. \\ Email: regragui.y@ucd.ac.ma
}

\author{
Najem Moussa \\ LAROSERI, Department of Computer Science \\ Chouaib Doukkali University, El Jadida Morocco. \\ Email: moussa.n@ucd.ac.ma
}

\begin{abstract}
There has been an increasing interest in the issue of real-time path planning based on the exchange of information about traffic conditions in vehicular ad hoc networks (VANETs). Because of the development in hardware, software, and communication technologies. However, real-time applications are facing several challenges for providing reliable communications (V2V and V2I) due to many effective factors that includes highspeed mobility and sparse vehicle density on the which increase the rate of topology change. In this paper, we first establish a real-time path planning application which utilizes real-time information about traffic conditions for providing shortest paths with minimum travel time on a weighted graph which is extracted from a real map. This application utilizes vehicular ad hoc networks (VANETs) to enable real-time communications among vehicles, roadside units (RSUs), and a central server. Each vehicle can request a shortest-path by sending a simple request packet to the server and waits for a reply. Finally, our path-planning method is implemented and simulated by using Veins framework (with IEEE 802.11 p/WAVE standard). Veins is capable of running OMNET++ and SUMO in parallel. Simulation results confirm that our proposed path-planning approach improves significantly the travel time.
\end{abstract}

Keywords-Ad hoc networks, Path planning, Mobility, Intelligent transportation system (ITS), Travel time, End-to-end delay.

\section{INTRODUCTION}

Intelligent transportation systems (ITS) have known a wide interest in academia and industry research. As important components of the ITS, VANETs are considered to be a promising concept for enabling numerous categories of applications to improve traffic safety, avoiding traffic moving congestion, and realtime applications in intelligent transportation systems [1].

Congestion problems are governed by several factors, among them the increase in the level of driving imperfections [2] which plays an important role in the formation of traffic jams within the transportation system, especially when it is combined with the increase in number of vehicles. Most often traffic jams are observed at roundabouts, crossings of roads, and crossings of highways [3]. When the density of cars increases, drivers' attention reduces and the imperfections in their driving styles are increasing. For example, a jam may appear just due to some traffic interweaves at roundabouts caused by imperfect driving styles.

In order to solve traffic related problems, several approaches are proposed, but most of them does not offer an efficient solution as they consider only the extending the road network. Another class of recent approaches have been proposed to solve this issue based on communication systems and navigation devices. These approaches usually use systems based on roadside and multi-hop communications between vehicles to get the traffic information and send the information to base station. Among the proposed solutions, path-planning schemes are expected to improve traffic conditions and achieve a globally optimal vehicle traffic as they efficiently allow collecting real-time information about traffic conditions by using V2V and V2I communications in VANETs. However, most existing approaches focus only on avoiding traffic conditions and neglect some prior issues such as drivers travel length or time. Thus, the first key issue for providing efficient and reliable services in transportation systems involves such proposed approach to take into consideration the travel time and length as key indicators of performance and efficiency.

To this end, we propose a real-time path-planning application that use VANETs communication to reduce travel time and path length. Our proposed path-planning strategy is based on the exchange of real-time traffic information about traffic conditions to plan navigation path dynamically. Our strategy is evaluated under different number of vehicles based on the analysis of travel time and end-to-end delay.

The rest of the paper is organized in the following way. We first review the related work in Section 2. Then we describe our path-planning strategy in section 3. Section 4 presents the simulation results and the paper is concluded in Section 5.

\section{RELATED WORK}

There have many attempts to solve the traffic congestion problems over the past several decades by extending the road network. However, most approaches does not satisfy the requirements due to limitation of resources (e.g., cost and places) and rapid increase in the number of cars. Thus, intelligent solutions, such as Vehicular Ad Hoc Networks (VANETs) are required to overcome this problem by considering efficient path-planning approach based on real-time exchange of information about traffic conditions. For example, the authors in [4] proposed a new method to dynamically calculate the current traveling time in a street by using RSUs at the start and end of each street. Efficiency of the proposed method is investigated in a realistic large scale city using a network simulator as a VANET scenario by employing SUMO, OMNET++ and 
VEINS. However, the authors dose not reported how vehicles triggers a broadcasts of the calculated travel time when it reaches the end of street. In [4], the authors proposed a realtime path-planning algorithm, which not only improves the overall spatial utilization of a road network but reduces average vehicle travel cost for avoiding vehicles from getting stuck in congestion as well. The proposed algorithm is based on a stochastic Lyapunov optimization technique which is exploited to address the globally optimal path-planning problem. The obtained results show that the proposed path-planning algorithm outperforms the traditional distributed path planning in terms of balancing the spatial utilization and drivers' travel cost. In [5] the authors introduced a novel dynamic vehicular path planning solution. The proposed solution does not rely on infrastructures to collect traffic information. Meanwhile, It utilizes density-speed traffic flow model to predict the traffic condition. In addition, a dynamic candidate path selection algorithm is developed to reduce the redundant data collection overhead. This approach has been extensively evaluated using large scale traffic trace based simulation have been performed. The obtained results show that the proposed approach outperforms some existing solutions in terms of communication efficiency and path planning effectiveness. In [6] the authors proposed a route planning algorithm to calculate the route with the shortest travelling time based on real-time traffic information by using VANETs. At each road segment, a travelling vehicle is able to exchange information about traffic information with other neighbor vehicles by using the IEEE 802.11p standard and GPS navigation application implemented on the Android platform. This approach uses the average recorded driving speed as an indicator of traffic state at road segments.

Another class of approaches is based on using pathplanning to reduce road traffic congestion and avoiding traffic jam in large cities. For example, in [7], to avoid traffic jam in large cities, the authors proposed an intelligent traffic system called CIDMERA, which improves the overall spatial utilization of a road network. CIDMERA also is able to reduce the average vehicle travel costs by avoiding vehicles from getting stuck in traffic. In [8] the authors proposed a distributed, collaborative traffic congestion detection and dissemination system that uses VANET. This system is based on the use of smart phone by each driver order to detect location through Geographic Position based System (GPS). This information is sent to a remote server which detects traffic congestion. Once congestion is confirmed the congestion information is disseminated to the end user phone through RSUs.

\section{MODEL DESCRIPTIONS}

Since the traffic dynamic is a based time variable that impacts the travel time in each road segment. In this paper, we present a complete design of a path-planning strategy capable of providing shortest paths in terms of travel time based on an efficient VANET environment. In our simulation scenario, each vehicle is equipped with an IEEE $802.11 \mathrm{p}$ compliant radio device, and implements the WAVE 1609.4 protocol for multi-channel protocol operations. Then, each vehicle in the network is able to exchange information about traffic conditions with other vehicles or RSUs. Based on the current state-of traffic flow in road segments, each vehicle crossing a road segment (i,j) records the start time and end
TABLE I. SUMMARY OF THE MATHEMATICAL NOTATIONS.

\begin{tabular}{|c|c|}
\hline Symbol & Description \\
\hline $\mathrm{V}$ & The set of vehicles in the network \\
\hline II & The set of all intersections \\
\hline $\mathbb{S}$ & The set of all road segments \\
\hline $\mathbb{R}$ & The set of all RSUs in the network \\
\hline $\mathbb{P}$ & The set of all packets in the network \\
\hline$n_{i} j$ & The number of vehicles in road segment $(\mathrm{i}, \mathrm{j})$ \\
\hline$T_{i}(t)$ & The travel time for vehicle $\mathrm{i}$ at time $\mathrm{t}$. \\
\hline $\operatorname{Re} f_{s}(i, j)$ & GPS reference point that indicates the start of road segment $(i, j)$. \\
\hline $\operatorname{Re} f_{e}(i, j)$ & GPS reference point that indicates the end of road segment $(\mathrm{i}, \mathrm{j})$. \\
\hline Start $_{t}(i, j)$ & The start time of vehicle's travel on road segment $(i, j)$. \\
\hline $\operatorname{End}_{t}(i, j)$ & The end time of vehicle's travel on road segment $(i, j)$. \\
\hline$T_{s}$ & Is the simulation time. \\
\hline$T_{i n t}$ & $\begin{array}{l}\text { The time interval for sending the average travel time for road } \\
\text { segments by the nearest RSU. }\end{array}$ \\
\hline
\end{tabular}

time based on the measurement of euclidian distance between vehicle position and GPS-based reference points that define the start and the end of road segment length. When vehicle $\mathrm{v}$ reaches the end of road segment $(i, j)$, it broadcast the travel time to a nearby RSU $r_{j}$ deployed just near to the end of this road segment (see Algorithm1).

By taking into consideration the travel time information that vehicles share with RSUs in the network, each RSU is the able to send these information to the server. The RSU collects the travel time information based on the road segment id and the type of packet indicated in the header of received packet. Based on these information, each RSU $r_{j}$ is able measure the average travel time per road segment. In addition, each RSU $r_{j}$ periodically broadcast a beacon to the server containing the average travel time for all nearby road segments $(i, j)$ (see Algorithm2). The received packets updates of travel times are treated by the server and recorded in the data base. By taking advantage of travel time information stored in the database, the server can establish a directed weighted graph (see Fig. 1), where each link represents a road segment in the considered map. Indeed, the server can automatically answer vehicles' requests by applying Bellman-Fords shortest path algorithm [9] (see Fig. 2). In this paper, only one vehicle is selected and assigned authorization to requests new routes from the server between edges ( 1 and 30 ). This vehicle is configured to travel several times between edge ( 1 and 30 or 30 and 1). Upon receiving a reply packet containing the new route, therefore the selected vehicle can update its trajectory based on received information from the server.

\section{A. Shortest path model}

Here, we aims to answer vehicles' requests to get the shortest path between a source and destination locations. the Solution used here is based on the Bellman-Fords shortest path algorithm [9], which has been widely used to solve several problems including network routing and optimal route planning in navigation systems.

In this paper, we use Bellman-Ford algorithm as a Shortest path algorithm by considering the measurement of travel time instead of the distance between edges in the graph. Using the following algorithm and based on measurement of the travel time in each road segment, Bellman-Ford algorithm can give a 
supplementary advantage because the distance can not reflect road traffic state, whereas the consideration of travel time can give us optimal prediction of road traffic state. The BellmanFord equation is given as:

$$
T_{t}(i, j)=\min _{k \in \text { intersections }}\left\{T_{t}(i, k)+T_{t}(k, j)\right\}
$$

where $T_{t}(i, k)$ is travel time for road segment $(\mathrm{i}, \mathrm{j}), \mathrm{i}$ and $\mathrm{j}$ denote intersection $\mathrm{i}$ and $\mathrm{j}$, respectively.

\section{Simulation ENVIRONMENT}

Different simulation environments were performed to evaluate the performance of our path-planning strategy. First, SUMO (Simulation of Urban Mobility) a microscopic road traffic simulation, is used to simulate the traffic dynamic in a real map. Second, VENS is a network simulator capable of simulation VANETS based on different modules specifically used to enable communication in a vehicular environment. This simulator is used with OMNET++ (Network simulator) and SUMO respectively. We evaluated our path-planning strategy

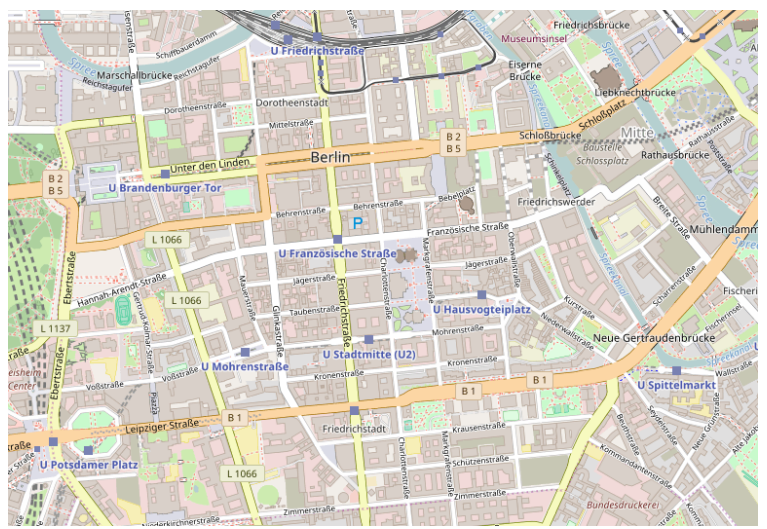

(a)
TABLE II. Network parameters used in the simulation.

\begin{tabular}{|l||l|}
\hline Parameter & Value \\
\hline \hline Roadmap size & $1620 \mathrm{~m} \times 1620 \mathrm{~m}$ \\
Simulation time & $2000 \mathrm{~s}$ \\
Beacon massage rate & 10 packet per second \\
Size of message & 500 bytes \\
MAC/PHY & IEEE $802.11 \mathrm{p} /$ WAVE \\
Obstacles & without \\
Number of vehicles & 5,160] \\
Mobility generator & SUMO \\
The model used for car following & carFollowModel \\
Lane change model & LC2013 \\
\hline
\end{tabular}

in VEINS based on IEEE $802.11 \mathrm{p} /$ WAVE standard under different car densities. In our scenario, we analyzed two main performance indicators, the end-to-end delay and the average travel time.

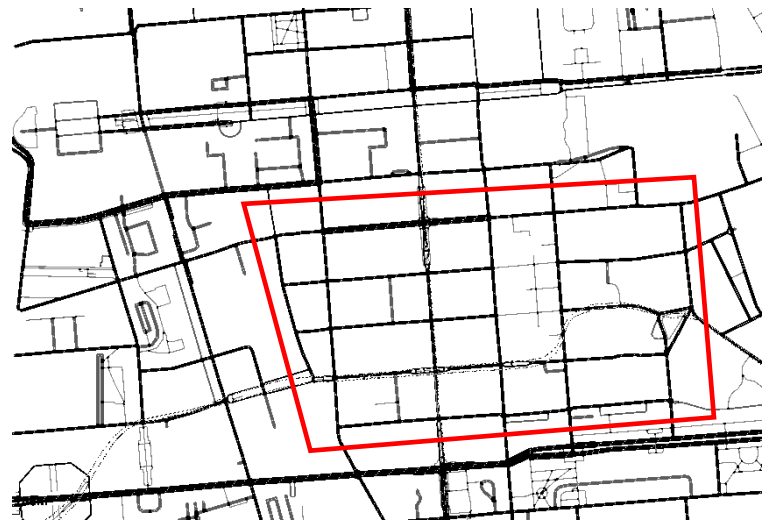

(b)

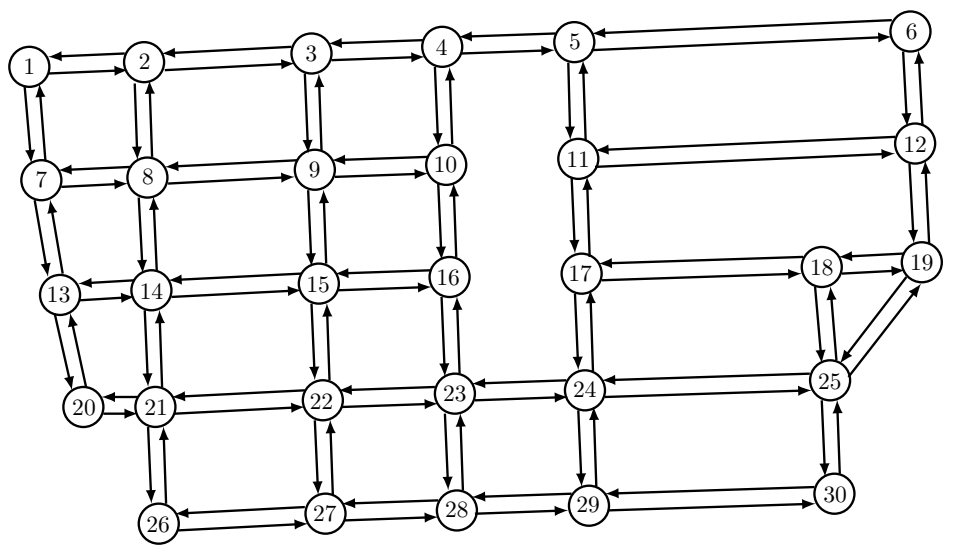

(c)

Fig. 1. Illustration of the considered traffic scenario: (a) Berlin map, (b) The simulation area and (b) The directed weighted graph extracted from the map. 


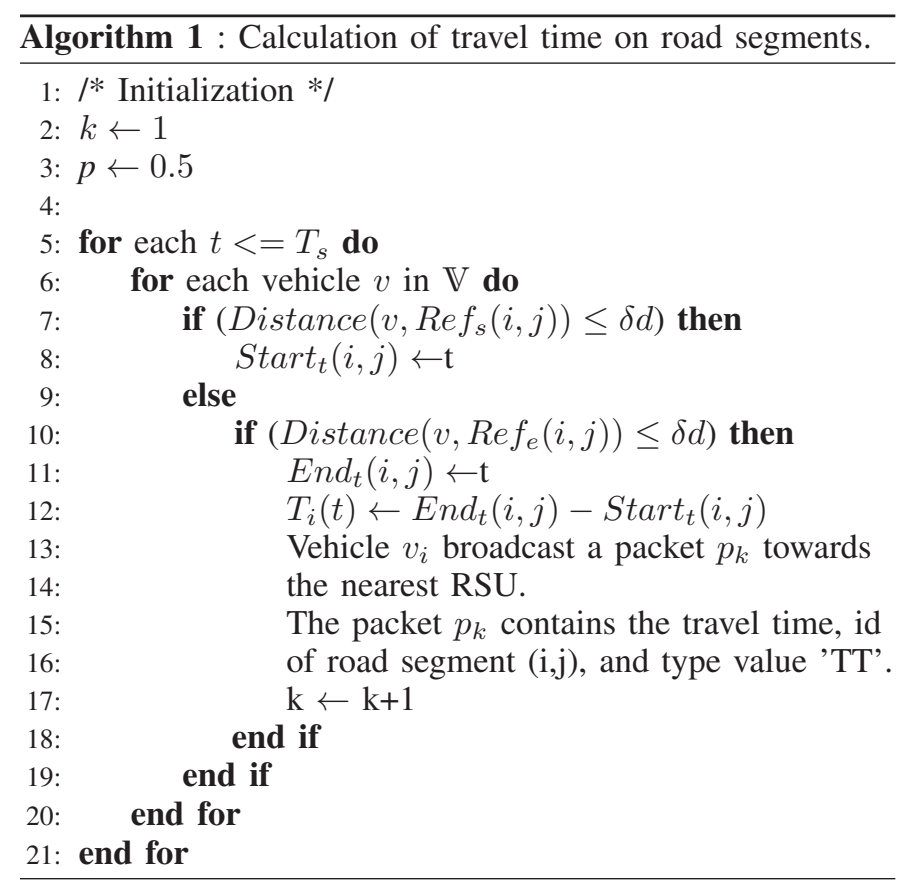

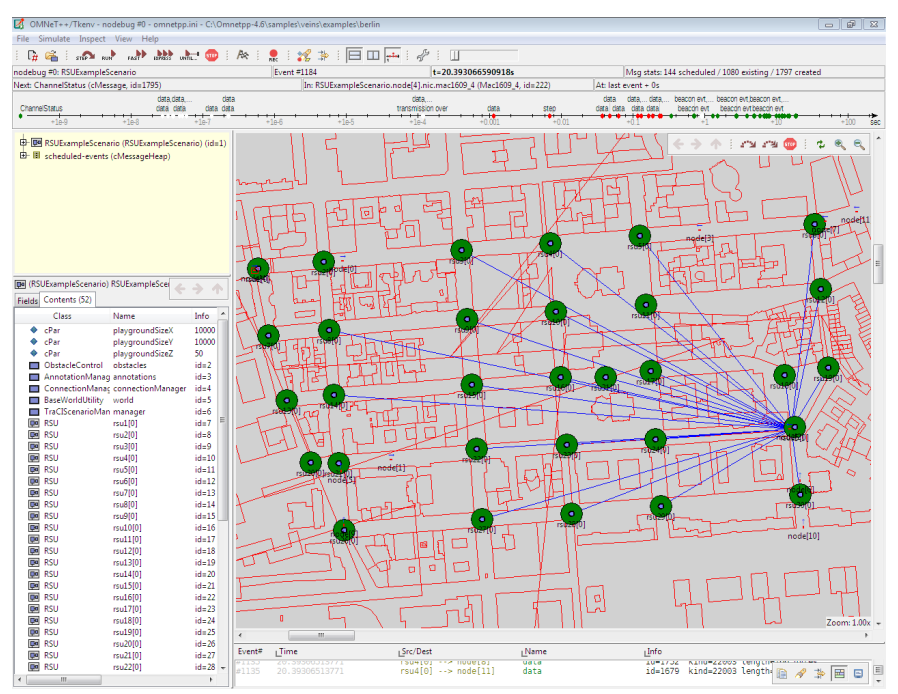

Fig. 3. Illustration example of our scenario with VEINS simulator.

\section{A. Performance Metrics}

Here we define two main performance metrics to evaluate the performance of our path-planning strategy. We monitor the change in the end-to-end delay and travel time for a considered vehicle traveling from source $\mathrm{S}$ toward destination $\mathrm{D}$. All the measured performance metrics considered in this work are described in details as follows.

- Average travel time: we define the arrival time as a sequence of time intervals which characterize the resulting time interval between injection time $\left(\mathbf{t}_{i n j}^{i}\right)$ when vehicle starts move from a source location and the arrival time $\left(\mathbf{t}_{a r}^{i}\right)$ when vehicle reach the destination location where $\mathbf{n}_{\text {travels }}$ is total number of vehicles' travels between $\mathrm{S}$ and $\mathrm{D}$. The travel time is defined as follows:

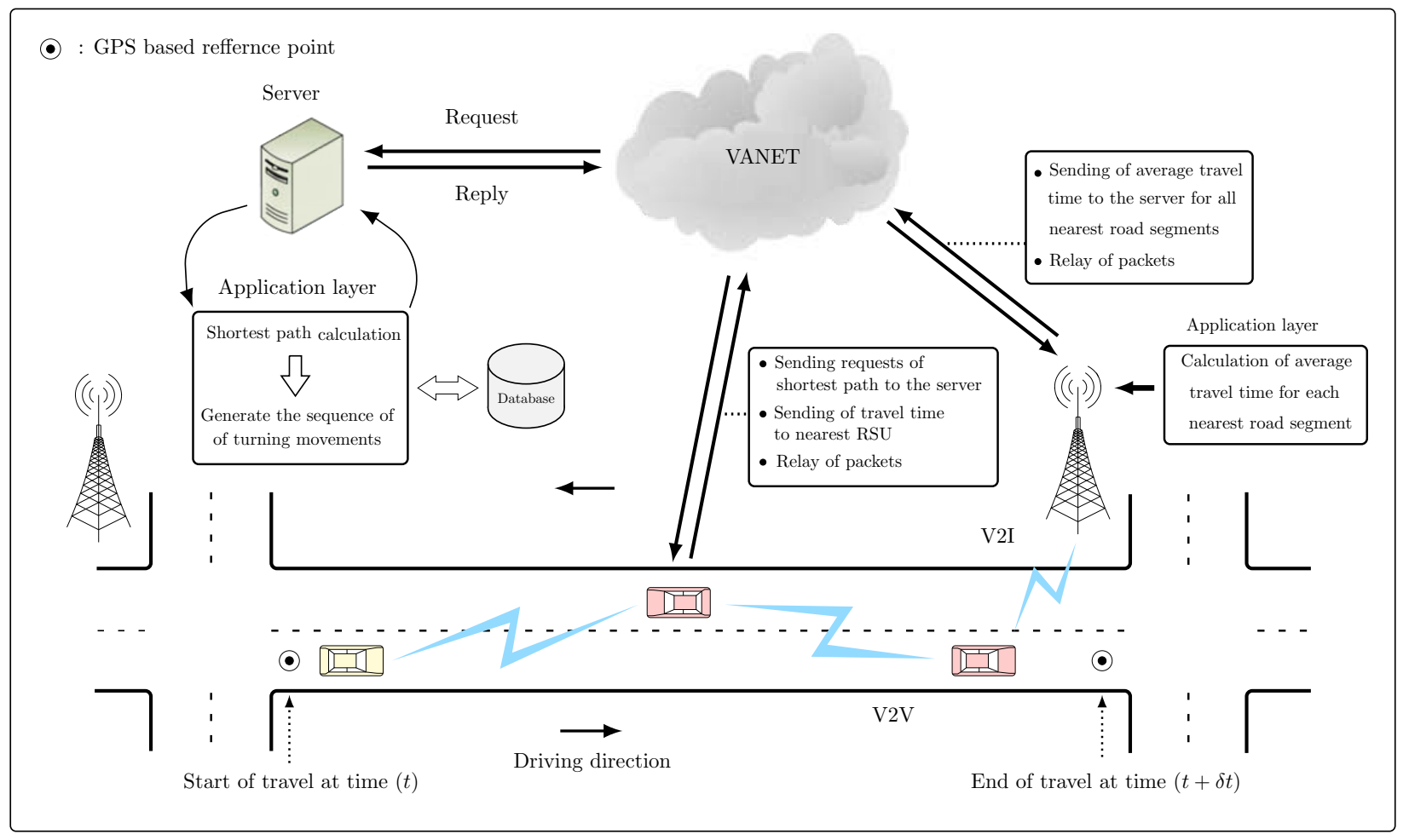

Fig. 2. Illustration of the interaction between all components considered in the VANET environment. 


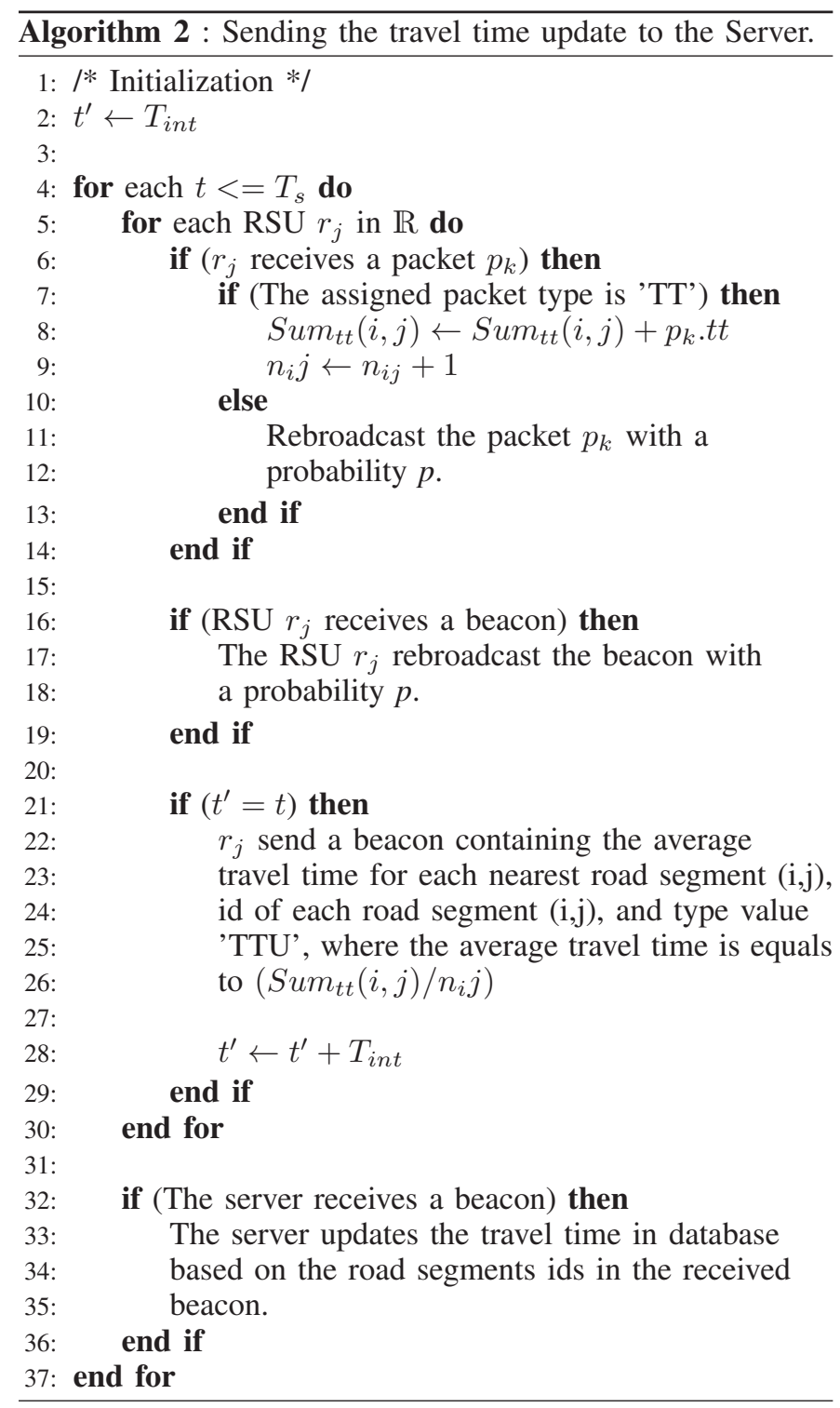

TABLE III. Attenuation, Physical Layer, and MAC.

\begin{tabular}{|l||l|}
\hline Parameter & Value \\
\hline \hline Parameter & Value \\
Frequency & $5.89 \mathrm{GHz}$ \\
Channel bandwidth & $10 \mathrm{MHz}$ \\
Bitrate rate & $18 \mathrm{Mbit} / \mathrm{s}$ \\
Transmit power & $20 \mathrm{~mW}$ \\
Sensitivity & $-89 \mathrm{dBm}$ \\
\hline
\end{tabular}

$$
\text { Average travel time }=\frac{\sum_{i=1}^{i=n_{\text {travals }}}\left(t_{a r}^{i}-t_{i n j}^{i}\right)}{n_{t r a j}}
$$

- Average end-to-end delay: we define the end-toend delay as a sequence of time interval which characterize the resulting time period including the data transmission delay, the propagation delay, the nodal processing delay, and the queuing delay at each intermediate node. We then calculate the end-to-end delay as the difference between the send time $\left(t_{\text {sen }}^{i}\right)$ and the received time $\left(t_{r e c}^{i}\right)$ of successfully received packet $\left(p k t_{r e c}^{i}\right)$ by the destination, where $n_{p k t_{r e c}}$ is total number of received packets. The average end-toend delay is defined as follows:

$$
\mathbf{E E D}=\frac{\sum_{i=1}^{i=n_{p k t_{r e c}}}\left(t_{r e c}^{i}-t_{\text {sen }}^{i}\right)_{p k t_{r e c}^{i}}}{n_{p k t_{r e c}}}
$$

\section{Simulation ANAlysis}

In this section, we present simulation results to illustrate the impact of number of vehicles on both travel time and the end to end delay. Also, the results show a comparison in terms of travel with and without strategy under varying number of vehicles. Fig. 5(a) shows that the travel time is decreased significantly when using our path-planning strategy. In contrast, when the path-planning strategy is not used, the travel time increases exponentially as function of number of vehicles. Fig. 5(b) shows that the end-to-end delay almost remains stable as function of number of vehicles, but suddenly the end-to-end delay increases when the number of vehicles reaches a higher value. Moreover, the reply packets possess an increased end-to-end delay as compared with that related to requests packets. Also, the results show that the increase in the number of vehicles ( $\mathrm{N}=160$ vehs) has a significant impact on the performance of network in terms of end-to-end delay, but not on the road segments' state as function of travel time, where the travel time per road segment is almost the same as compared with that in the case of low number of vehicles ( $\mathrm{N}=40$ vehs) (see Fig. 4). We can explain that by the fact that when the selected vehicle sends a request to the server, the request high delays due to increased interference between more probable senders in the same zone, which causes a degradation of the reachability of requests or reply. This situation lead to the so-called broadcast storm due to an increase in the number of hidden node and exposed terminal. Accordingly, in our scenario, vehicle and RSUs rebroadcast the received packet with a probability $(p=0.5)$. This means that the increases in the rebroadcasting probability may amplify the problem. In addition, loss of requests or reply means that any delayed reply will received is not important as the vehicle' trajectory is changed. Based on these results, we expect that when our strategy is combined with an efficient broadcast dissemination scheme, it will gives a supplementary good results in terms of travel time, but this is left as future work.



Fig. 4. Comparison of travel time per road segment under two different number of vehicles. 


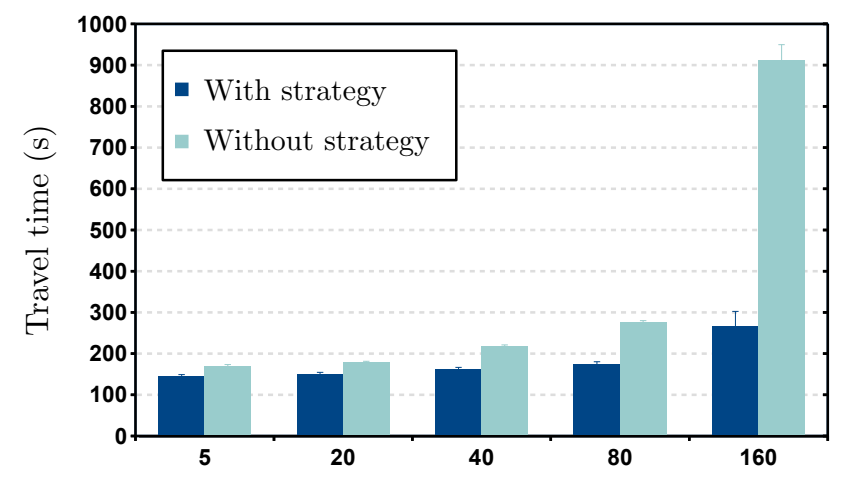

(a)

Number of vehicles

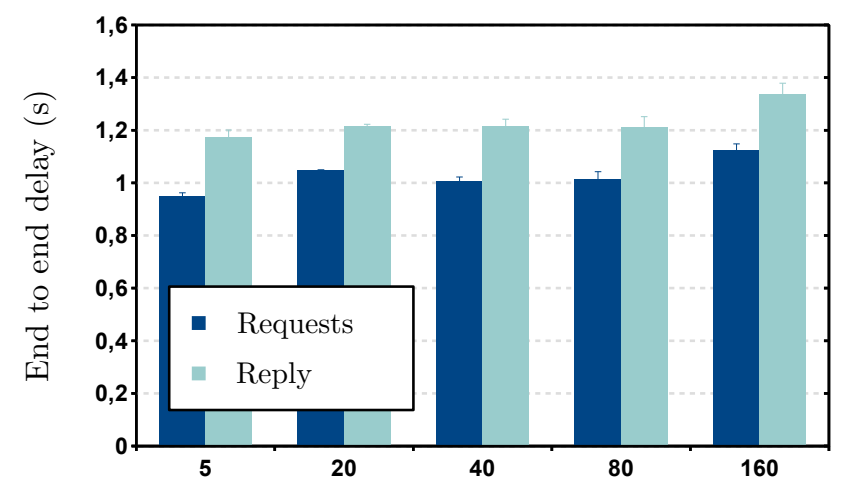

(b)

Number of vehicles
Wireless Communications, IEEE, vol. 13, no. 5, pp. 22-28, 2006.

[2] F. Járai-Szabó and Z. Néda, "Earthquake model describes traffic jams caused by imperfect driving styles," Physica A: Statistical Mechanics and its Applications, vol. 391, no. 22, pp. 5727-5738, 2012.

[3] A. Schadschneider, "Traffic flow: a statistical physics point of view," Physica A: Statistical Mechanics and its Applications, vol. 313, no. 1-2, pp. 153-187, 2002.

[4] H. Noori and M. Valkama, "Impact of vanet-based v2x communication using ieee $802.11 \mathrm{p}$ on reducing vehicles traveling time in realistic large scale urban area," in Connected Vehicles and Expo (ICCVE), 2013 International Conference on. IEEE, 2013, pp. 654-661.

[5] Z. He, J. Cao, and T. Li, "Mice: A real-time traffic estimation based vehicular path planning solution using vanets," in Connected Vehicles and Expo (ICCVE), 2012 International Conference on. IEEE, 2012, pp. $172-178$.

[6] H. Yu, J. Yoo, and S. Ahn, "A vanet routing based on the real-time road vehicle density in the city environment," in Ubiquitous and Future Networks (ICUFN), 2013 Fifth International Conference on. IEEE, 2013, pp. 333-337.

[7] A. M. de Souza, R. S. Yokoyama, G. Maia, A. Loureiro, and L. Villas, "Real-time path planning to prevent traffic jam through an intelligent transportation system," in Computers and Communication (ISCC), 2016 IEEE Symposium on. IEEE, 2016, pp. 726-731.

[8] C. Jayapal and S. S. Roy, "Road traffic congestion management using vanet," in Advances in Human Machine Interaction (HMI), 2016 International Conference on. IEEE, 2016, pp. 1-7.

[9] A. Orda and R. Rom, "Shortest-path and minimum-delay algorithms in networks with time-dependent edge-length," Journal of the ACM (JACM), vol. 37, no. 3, pp. 607-625, 1990.

Fig. 5. Effect of increasing the number of vehicles: (a) Travel time and (b) End-to-end delay.

\section{CONCLUSION}

We have introduced a novel path-planning strategy based on multi-hop V2V and V2I communications. This strategy uses a short path algorithm based on the computation of the travel time in each road segment. After collecting the average travel time for each road segment, the RSUs send beacons to the server located in the middle of the simulation area. The server can answers requests of vehicles by sending a reply that contains a new route. The simulation results show that our strategy is efficient to provide reliable routes under different car densities. In the absence of a path-planning strategy, the travel time highly increases as compared with that illustrated by our strategy. Furthermore, we have found that the number of vehicles has impact on the reachability and the end-to-end delay for both requests and reply packets. The lack of reachability and the increase in the end-to-end delay is related phenomenon of the so-called broadcast storm due to an increase in the number of hidden node and exposed terminal. With supplementary effort, our strategy can be used with other efficient broadcast dissimilation scheme such as Weighted p-Persistence or Slotted p-Persistence. Future work could include simulation scenario with confident broadcast dissimilation scheme and other improvements.

\section{REFERENCES}

[1] T. Kosch, C. J. Adler, S. Eichler, C. Schroth, and M. Strassberger, "The scalability problem of vehicular ad hoc networks and how to solve it," 